\title{
Pacific
}

Journal of

Mathematics

\section{ON A LIU-YAU TYPE INEQUALITY FOR SURFACES}

Oussama Hijazi, Sebastián Montiel and Simon Raulot 


\title{
ON A LIU-YAU TYPE INEQUALITY FOR SURFACES
}

\author{
Oussama Hijazi, Sebastián Montiel and Simon Raulot
}

Let $\Omega$ be a compact mean-convex domain with smooth boundary $\Sigma:=\partial \Omega$, in an initial data set $\left(M^{3}, g, K\right)$, which has no apparent horizon in its interior. If $\Sigma$ is spacelike in a spacetime $\left(\mathscr{E}^{4}, g_{\mathscr{E}}\right)$ with spacelike mean curvature vector $\mathscr{H}$ such that $\Sigma$ admits an isometric and isospin immersion into $\mathbb{R}^{3}$ with mean curvature $H_{0}$, then

$$
\int_{\Sigma}|\mathscr{H}| d \Sigma \leq \int_{\Sigma} \frac{H_{0}^{2}}{|\mathscr{H}|} d \Sigma
$$

If equality occurs, we prove that there exists a local isometric immersion of $\Omega$ in $\mathbb{R}^{3,1}$ (the Minkowski spacetime) with second fundamental form given by $K$. We also examine, under weaker conditions, the case where the spacetime is the $(n+2)$-dimensional Minkowski space $\mathbb{R}^{n+1,1}$ and establish a stronger rigidity result.

\section{Introduction}

Let $\left(\mathscr{E}^{4}, g_{\mathscr{E}}\right)$ be a spacetime satisfying the Einstein field equations; that is, $\left(\mathscr{E}^{4}, g_{\mathscr{E}}\right)$ is a 4-dimensional time-oriented Lorentzian manifold such that

$$
\operatorname{Ric}_{\mathscr{E}}-\frac{1}{2} R_{\mathscr{E}} g_{\mathscr{E}}=\mathscr{T}
$$

where $R_{\mathscr{E}}$ (respectively, $\mathrm{Ric}_{\mathscr{E}}$ ) denotes the scalar curvature (respectively, the Ricci curvature) of $\left(\mathscr{E}, g_{\mathscr{E}}\right)$, and $\mathscr{T}$ is the energy-momentum tensor which describes the matter content of the ambient spacetime. We also assume that $\left(\mathscr{E}^{4}, g_{\mathscr{E}}\right)$ satisfies the dominant energy condition; that is, its energy-momentum tensor $\mathscr{T}$ has the property that, for every future-directed causal vector $\eta \in \Gamma\left(T_{\mathscr{E}}^{\mathscr{C}}\right)$, the vector field dual to the one-form $-\mathscr{T}(\eta, \cdot)$ is a future-directed causal vector of $T \mathscr{E}$.

Let $M^{3}$ be an immersed spacelike hypersurface of $\left(\mathscr{E}^{4}, g_{\mathscr{E}}\right)$ with induced Riemannian metric $g$. Assume that $T$ is the future-directed timelike normal vector to $M$ and denote by $K$ the associated second fundamental form defined by $K(X, Y)=g_{\mathscr{E}}\left(\nabla_{X}^{\mathscr{E}} T, Y\right)$ for all $X, Y \in \Gamma(T M)$. Here $\nabla^{\mathscr{E}}$ denotes the Levi-Civita

S. Montiel was partially supported by the Spanish MEC-FEDER grant no. MTM2011-22547. MSC2010: 53C27, 53C40, 53C 80.

Keywords: manifolds with boundary, Dirac operator, Einstein equations, initial data set, mean curvature, holographic principle. 
connection of the spacetime. Then the Gauss, Codazzi and Einstein equations provide constraint equations on $M$, given by

$$
\left\{\begin{array}{l}
\mu=\frac{1}{2}\left(R-|K|_{M}^{2}+\left(\operatorname{tr}_{M}(K)\right)^{2}\right), \\
J=-\delta\left(K-\operatorname{tr}_{M}(K) g\right)
\end{array}\right.
$$

where $R$ is the scalar curvature of $\left(M^{3}, g\right),|K|^{2}$ and $\operatorname{tr}(K)$ denote the squared norm and the trace of $K$ with respect to $g$, and $\delta$ is the divergence on $M$. Here $\mu$ and $J$ are the energy and momentum density of the matter fields, and are given by

$$
\mu=\mathscr{T}(T, T) \quad \text { and } \quad J_{i}=\mathscr{T}\left(e_{i}, T\right)
$$

for $1 \leq i \leq 3$, where $\left\{e_{1}, e_{2}, e_{3}\right\}$ is a local basis of the spatial tangent space of $M$. The dominant energy condition for the spacetime implies that $\mu \geq|J|$ as functions on $M$. A triplet $\left(M^{3}, g, K\right)$ which satisfies the dominant energy condition is called an initial data set.

Now we consider a codimension-two spacelike orientable surface $\Sigma^{2}$ in the spacetime $\mathscr{E}^{4}$. We will represent by $\mathscr{H}$ the mean curvature vector field on $\Sigma^{2}$, defined as

$$
\mathscr{H}=\operatorname{tr} I I,
$$

where $I I$ is the second fundamental form of this immersion. Since the normal space at each point of $\Sigma^{2}$ is a Lorentzian plane, it can be spanned by two futuredirected null normal vector fields $\mathcal{N}_{+}$and $\mathcal{N}_{-}$, normalized in such a way that $\left\langle\mathcal{N}_{+}, \mathcal{N}_{-}\right\rangle=-\frac{1}{2}$. We denote by $\theta_{+}$and $\theta_{-}$the components of $\mathscr{H}$ with respect to $\mathcal{N}_{+}$and $\mathcal{N}_{-}$. They are the so-called future-directed null expansions of $\mathscr{H}$, and measure the area growth when $\Sigma^{2}$ varies in the corresponding directions. It is clear that

$$
|\mathscr{H}|^{2}=-\theta_{+} \theta_{-} .
$$

If $\theta_{+}$and $\theta_{-}$are both negative, the surface will be called a trapped surface. A surface with $\theta_{+}=0$ or $\theta_{-}=0$ is called an apparent horizon (or a marginally trapped surface). Note that if $\Sigma^{2}$ is trapped or marginally trapped, then the mean curvature vector $\mathscr{H}$ is a causal vector at each point. This is why the mean curvature field $\mathscr{H}$ being spacelike everywhere is equivalent to $\Sigma$ being an untrapped surface.

In the case that $\Sigma^{2}$ spans a spacelike hypersurface in the spacetime, that is, when there exists a spacelike hypersurface $\Omega^{3}$ immersed in $\mathscr{E}^{4}$ such that $\partial \Omega^{3}=\Sigma^{2}$, the normal null vector fields $\mathcal{N}_{+}$and $\mathcal{N}_{-}$may be ordered in such a way that they project onto directions tangent to $\Omega^{3}$ which are, respectively, outer and inner normal at each point of $\Sigma^{2}$. In other words, if $N$ is the inner normal unit vector field on $\Sigma^{2}$ tangent to $\Omega^{3}$ and $T$ is the future-directed timelike normal to $\Omega^{3}$ in $\mathscr{E}^{4}$, we put

$$
\mathcal{N}_{+}=\frac{1}{2}(T-N) \quad \text { and } \quad \mathcal{N}_{-}=\frac{1}{2}(T+N) .
$$


The second fundamental form of $\Sigma^{2}$ in $\mathscr{E}^{4}$ is given in terms of the Lorentzian basis of the normal bundle the hypersurface $\Omega$ by

$$
I I(X, Y)=g(A X, Y) N+g(B X, Y) T
$$

for all $X, Y \in \Gamma(T \Sigma)$, where $A X:=-\nabla_{X} N$ denotes the shape operator of $\Sigma^{2}$ in $\Omega^{3}$ and $\nabla$ is the Levi-Civita connection of the Riemannian metric $g$ on $M$. The mean curvature vector field $\mathscr{H}$ of $\Sigma$ in $\mathscr{E}$ can be reexpressed by

$$
\mathscr{H}=\theta_{+} \mathcal{N}_{-}+\theta_{-} \mathcal{N}_{+}=H N+\operatorname{tr}_{\Sigma}(K) T,
$$

where $H=\operatorname{tr} A$ is the mean curvature of $\Sigma^{2}$ in $\Omega^{3}$ and $\operatorname{tr}_{\Sigma}(K)$ is the trace on $\Sigma^{2}$ of the shape operator $K$ of $\Omega^{3}$ in $\mathscr{E}^{4}$. The norm of $\mathscr{H}$ can be also reexpressed as

$$
|\mathscr{H}|^{2}=H^{2}-\operatorname{tr}_{\Sigma}(K)^{2}=-\theta_{+} \theta_{-},
$$

where $\theta_{ \pm}=\operatorname{tr}_{\Sigma}(K) \pm H$ are the future-directed null expansions of $\mathscr{H}$. The spacelike surfaces with $\theta_{+}<0$ (respectively, $\theta_{-}<0$ ) are referred to as outer (respectively, inner) trapped surfaces. It is easy to see that untrapped submanifolds, that is, codimension-two spacelike submanifolds of a spacetime with spacelike mean curvature vector field, naturally divide into two disjoint classes:

Lemma 1. Let $\Sigma^{2}$ be a compact spacelike codimension-two submanifold embedded in a spacetime $\mathscr{E}^{4}$. Suppose that its mean curvature vector field $\mathscr{H}$ is spacelike and that $\Sigma^{2}$ is the boundary of a spacelike hypersurface $\Omega^{3}$ in $\mathscr{E}^{4}$. Then $\Omega^{3}$ is either mean-convex or mean-concave.

Proof. It suffices to take into account that if $\left(\theta_{+}, \theta_{-}\right)$are the future-directed null expansions of the mean curvature vector field $\mathscr{H}$ associated to the embedding of $\Sigma^{2}$ in the domain $\Omega^{3}$, we have, from (1),

$$
0<|\mathscr{H}|^{2}=-\theta_{+} \theta_{-} \text {and } \theta_{+}-\theta_{-}=2 H,
$$

where $H$ is the inner mean curvature function of $\Sigma^{2}$ in $\Omega^{3}$. The first of these two equalities implies that $\theta_{+}$and $\theta_{-}$have opposite signs everywhere on $\Sigma^{2}$. Then, from the second one, we have that either $H>0$ or $H<0$ on the whole of $\Sigma^{2}$.

Note that this fact obviously holds for higher-dimensional initial data sets. In the following, an untrapped surface (respectively, a codimension-two untrapped submanifold) which bounds a compact, connected and mean-convex spacelike hypersurface will be referred to as an outer untrapped surface (respectively, an outer untrapped submanifold). It is worth noting that round spheres in Euclidean slices are untrapped surfaces. The same occurs in general for large radial spheres in asymptotically flat spacelike hypersurfaces.

We now give the precise statement of our main result: 
Theorem 2. Let $\Omega$ be a compact domain with an outer untrapped boundary surface $\Sigma:=\partial \Omega$ in an initial data set $\left(M^{3}, g, K\right)$. If $\Omega$ has no apparent horizon in its interior, then for all $\varphi \in \Gamma(\$ \Phi)$,

$$
\int_{\Sigma}\left(\frac{1}{|\mathscr{H}|}|\not D \varphi|^{2}-\frac{|\mathscr{H}|}{4}|\varphi|^{2}\right) d \Sigma \geq 0
$$

where $\$ \Sigma$ is the extrinsic spinor bundle on $\Sigma$ and $\not D$ is the extrinsic Dirac operator (see Section 2). Moreover, if equality occurs, then there exists a local isometric immersion of $\Omega$ in $\mathbb{R}^{3,1}$ with $K$ as second fundamental form.

As a direct application, we prove the following result:

Theorem 3. Under the conditions of Theorem 2, assume furthermore that $\Sigma$ admits an isometric and isospin immersion into $\mathbb{R}^{3}$ with mean curvature $H_{0}$. Then

$$
\int_{\Sigma}|\mathscr{H}| d \Sigma \leq \int_{\Sigma} \frac{H_{0}^{2}}{|\mathscr{H}|} d \Sigma
$$

Moreover, if equality occurs, then $\Sigma$ is connected and there exists a local isometric immersion of $\Omega$ in $\mathbb{R}^{3,1}$ with second fundamental form given by $K$ and mean curvature vector of $\Sigma$ satisfying $|\mathscr{H}|=H_{0}$.

If we consider the case of codimension-two outer untrapped submanifolds in the $(n+2)$-dimensional Minkowski spacetime $\mathbb{R}^{n+1,1}$, we prove that we can remove the assumption on the nonexistence of apparent horizons (see Theorem 14). Moreover, in this situation, we completely characterize the equality case. Namely:

Theorem 4. Let $\Sigma$ be a codimension-two outer untrapped submanifold in $\mathbb{R}^{n+1,1}$. If $\Sigma$ admits an isometric and isospin immersion into $\mathbb{R}^{n+1}$ with mean curvature $H_{0}$, then inequality (3) holds and equality is achieved if and only if $\Sigma$ lies in a hyperplane in $\mathbb{R}^{n+1,1}$ and $\Sigma$ is connected.

Remark 5. In Theorems 3 and 4, we assumed that the boundary hypersurface of a compact domain in a certain spin manifold admits an isospin immersion into a Euclidean space. In general, an $(n+1)$-dimensional spin manifold induces a spin structure on each of its orientable immersed hypersurfaces through their corresponding immersions (see Section 2.2 below). Two distinct immersions of an orientable manifold $\Sigma^{n}$ into two (possibly different) $(n+1)$-dimensional spin manifolds are said to be isospin when the spin structures induced on $\Sigma^{n}$ from the corresponding ambient manifolds coincide (up to an equivalence). Recall that spin structures on $\Sigma^{n}$ are parametrized by the cohomology group $H^{1}\left(\Sigma^{n}, \mathbb{Z}_{2}\right)$. Thus, for example, if $\Sigma^{n}$ is a simply connected manifold, any two immersions of $\Sigma^{n}$ in two arbitrary $(n+1)$-dimensional spin manifolds must be isospin. Consequently if the surface $\Sigma$ in Theorem 3 has genus zero or the hypersurface $\Sigma$ in Theorem 4 is 
simply connected, we only need to suppose that they are mean-convex in their initial data sets and that they can be immersed as hypersurfaces in a Euclidean space.

Also it is clear that when the two immersions defined on $\Sigma^{n}$ lie in the same ambient space and are regularly homotopic, the associated induced spin structures are equivalent. In fact, two immersions are said to be regularly homotopic (isotopic, according to Pinkall [1985] and others) if we may pass continuously from one to the other through a family of immersions. Consequently they determine the same class in $H^{1}\left(\Sigma^{n}, \mathbb{Z}_{2}\right)$. Indeed in the case $n=2$, two spin structures induced from the spin structure of the 3-dimensional spin ambient space through two different embeddings are equivalent if and only if they are regularly homotopic (besides the previous reference, see [Hass and Hughes 1985, pp. 104-105] and [Benedetti and Silhol 1995, p. 656]).

Then take any compact mean-convex surface $\Sigma$ embedded in $\mathbb{R}^{3}$. This surface bounds a compact domain in three-dimensional Euclidean space which is a totally geodesic initial data set in the Minkowski space $\mathbb{R}^{3,1}$. If we slightly deform this surface, the positivity of the mean curvature is preserved by continuity, and, from the arguments above, the same holds for the induced spin structure. So there are examples of mean-convex boundaries in initial data sets of spacetimes admitting isospin immersions in Euclidean spaces. Many of them are nonconvex. In fact, take $\Sigma$ to be, for instance, a right cylinder with two half-spheres closing its extremes (after smoothing) or a torus of revolution thin enough (if we want to have some point with negative Gauss curvature).

Note that if $\Sigma$ is not convex, we cannot use the Weyl theorem and so we do not know whether it is possible to immerse $\Sigma$ isometrically in Euclidean space $\mathbb{R}^{3}$. This is why in this case, Theorems 3 and 4 should be viewed as comparison theorems for the mean curvatures of two immersions in the spirit of a classical result by Herglotz. Indeed, Herglotz [1943] gave a succinct proof of Cohn-Vossen's rigidity result for convex surfaces based on an integral inequality involving the second fundamental forms of two embeddings (see, e.g., [Montiel and Ros 1997, Section 7.4]). Our Theorem 3 provides an inequality of this type which could be a first step in enlarging the Cohn-Vossen theorem to include Euclidean mean-convex compact surfaces.

In this direction, one can easily see that Theorem 4 implies that the integral of the mean curvature is preserved through bendings of compact mean-convex hypersurfaces embedded in a Euclidean space. This was first proved by Almgren and Rivin [1998] (see also [Rivin and Schlenker 1999]).

Recall that Liu and Yau [2006] (see also [Liu and Yau 2003]) proved the following positivity result: Let $\left(\Omega^{3}, g, K\right)$ be an initial data set for the Einstein equation. Suppose that the boundary $\partial \Omega$ has finitely many components $\Sigma_{i}, 1 \leq i \leq l$, each of which has positive Gauss curvature and spacelike mean curvature vector in the spacetime. Then for all $i$, 


$$
\int_{\Sigma_{i}}|\mathscr{H}| d \Sigma \leq \int_{\Sigma_{i}} H_{0} d \Sigma
$$

Moreover, if equality occurs for some $i \in\{1, \ldots, l\}$, then $\partial \Omega$ is connected and the spacetime is flat along $\Omega$.

The proof of this result relies on a generalized version of the positive mass theorem and on the resolution of the Jang equation. One of the key ingredients in the proof is provided by the Weyl embedding theorem [1916], which asserts that the condition that $\Sigma$ embeds isometrically as a strictly convex hypersurface in $\mathbb{R}^{3}$ is equivalent to $\Sigma$ having positive Gauss curvature. Note that by the Cauchy-Schwarz inequality, inequality (4) implies (3).

More recently, Eichmair, Miao and Wang [Eichmair et al. 2012] generalized inequality (4) for time-symmetric initial data under weaker convexity assumptions for the embedding of $\Sigma$ in $\mathbb{R}^{3}$. We point out that, in contrast to Liu and Yau's result, we do not assume that the immersion is a strictly convex embedding. In particular, the mean curvature $H_{0}$ is not assumed to be positive.

\section{The Riemannian setting}

2.1. Preliminaries on spin manifolds. Let $(M, g)$ be an $(n+1)$-dimensional Riemannian spin manifold, which we will suppose from now on to be connected, and denote by $\nabla$ the Levi-Civita connection on its tangent bundle $T M$. We choose a spin structure on $M$ and consider the corresponding spinor bundle $S M$, a rank- $2^{(n+1) / 2}$ complex vector bundle. Denote by $\gamma$ the Clifford multiplication

$$
\gamma: \mathbb{C} \ell(M) \longrightarrow \operatorname{End}(\mathbb{S} M)
$$

which is a fiber-preserving algebra morphism. Then $\mathbb{S} M$ becomes a bundle of complex left modules over the Clifford bundle $\mathbb{C} \ell(M)$ over the manifold $M$. When $(n+1)$ is even, the spinor bundle splits into the direct sum of the positive and negative chiral subbundles:

$$
\mathbb{S} M=\mathbb{S} M^{+} \oplus \mathbb{S} M^{-},
$$

where $\mathbb{S} M^{ \pm}$are defined to be the \pm 1 -eigenspaces of the endomorphism $\gamma\left(\omega_{n+1}\right)$, with $\omega_{n+1}=i^{(n+2) / 2} e_{1} e_{2} \cdots e_{n+1}$ the complex volume form.

On the spinor bundle $\mathbb{S} M$, one has (see [Lawson and Michelsohn 1989]) a natural Hermitian metric, denoted by $\langle\cdot, \cdot\rangle$, and the spinorial Levi-Civita connection $\nabla$ acting on spinor fields. It is well-known that the Hermitian scalar product, the Levi-Civita connection $\nabla$ and the Clifford multiplication (5) satisfy, for any spinor fields $\psi, \varphi \in \Gamma(\mathbb{S} M)$ and any tangent vector fields $X, Y \in \Gamma(T M)$, the compatibility conditions 


$$
\begin{aligned}
\langle\gamma(X) \psi, \gamma(X) \varphi\rangle & =|X|^{2}\langle\psi, \varphi\rangle, \\
X\langle\psi, \varphi\rangle & =\left\langle\nabla_{X} \psi, \varphi\right\rangle+\left\langle\psi, \nabla_{X} \varphi\right\rangle, \\
\nabla_{X}(\gamma(Y) \psi) & =\gamma\left(\nabla_{X} Y\right) \psi+\gamma(Y) \nabla_{X} \psi .
\end{aligned}
$$

Since $\nabla \omega_{n+1}=0$, for $n+1$ even, the decomposition (6) is orthogonal and $\nabla$ preserves this decomposition.

The Dirac operator $D$ on $\mathbb{S} M$ is the first-order elliptic differential operator locally given by

$$
D=\sum_{i=1}^{n+1} \gamma\left(e_{i}\right) \nabla_{e_{i}},
$$

where $\left\{e_{1}, \ldots, e_{n+1}\right\}$ is a local orthonormal frame of $T M$. When $(n+1)$ is even, the Dirac operator interchanges positive and negative spinor fields; that is,

$$
D: \Gamma\left(\mathbb{S} M^{ \pm}\right) \longmapsto \Gamma\left(\mathbb{S} M^{\mp}\right) .
$$

2.2. Hypersurfaces and induced structures. In this section, we compare the restrictions $\$ \Phi \Sigma$ of the spinor bundle $\mathbb{S} M$ of a spin manifold $M$ to an orientable hypersurface $\Sigma$ immersed into $M$, and its Dirac-type operator $\not D$ to the intrinsic spinor bundle $\mathbb{S} \Sigma$ of the induced spin structure on $\Sigma$ and its fundamental Dirac operator $D_{\Sigma}$. A fundamental case will be when the hypersurface $\Sigma$ is just the boundary $\partial M$ of a manifold $M$. These facts are in general well-known (see, for example, [Bureš 1993; Trautman 1995; Bär 1998; Baum et al. 1990; Hijazi et al. 2001a; 2001b; 2002; Hijazi and Montiel 2014]). For completeness, we introduce the notation and key facts.

Denote by $\not$ the Levi-Civita connection associated with the induced Riemannian metric on $\Sigma$. The Gauss formula says that

$$
\nabla_{X} Y=\nabla_{X} Y-g(A X, Y) N,
$$

where $X, Y$ are vector fields tangent to the hypersurface $\Sigma$, the vector field $N$ is a global unit field normal to $\Sigma$, and $A$ stands for the shape operator corresponding to $N$; that is,

$$
\nabla_{X} N=-A X \text { for all } X \in \Gamma(T \Sigma) \text {. }
$$

We have that the restriction

$$
\$ \Sigma:=\left.\mathbb{S} M\right|_{\Sigma}
$$

is a left module over $\mathbb{C} \ell(\Sigma)$ for the induced Clifford multiplication

$$
\gamma: \mathbb{C} \ell(\Sigma) \longrightarrow \operatorname{End}(\$ \Phi)
$$


given by

$$
\gamma(X) \psi=\gamma(X) \gamma(N) \psi
$$

for every $\psi \in \Gamma(\$ \Phi)$ and $X \in \Gamma(T \Sigma)$. (Note that a spinor field on the ambient manifold $M$ and its restriction to the hypersurface $\Sigma$ will be denoted by the same symbol.) Consider the Hermitian metric $\langle\cdot, \cdot\rangle$ on $\$ \Sigma$ induced from that of $\mathbb{S} M$. This metric immediately satisfies the compatibility condition (7) if one considers the Riemannian metric on $\Sigma$ induced from $M$ and the Clifford multiplication $\gamma$ defined in (12). Now the Gauss formula (10) implies that the spin connection $\not$ on $\$ \Sigma$ is given by the spinorial Gauss formula

$$
\not_{X} \psi=\nabla_{X} \psi-\frac{1}{2} \gamma(A X) \psi=\nabla_{X} \psi-\frac{1}{2} \gamma(A X) \gamma(N) \psi
$$

for every $\psi \in \Gamma(\$ \Sigma)$ and $X \in \Gamma(T \Sigma)$. Note that the compatibility conditions (7), (8) and (9) are satisfied by $(\$ \Sigma, \gamma,\langle\cdot, \cdot\rangle, \not)$.

Denote by $\not D: \Gamma(\$ \Sigma) \rightarrow \Gamma(\$ \Phi)$ the Dirac operator associated with the Dirac bundle $\$ \Sigma$ over the hypersurface. It is a well-known fact that $\not D$ is a first-order elliptic differential operator which is formally $L^{2}$-selfadjoint. By (13), for any spinor field $\psi \in \Gamma(\mathbb{S} M)$,

$$
\not D \psi=\sum_{j=1}^{n} \gamma\left(e_{j}\right) \not{ }_{e_{j}} \psi=\frac{1}{2} H \psi-\gamma(N) \sum_{j=1}^{n} \gamma\left(e_{j}\right) \nabla_{e_{j}} \psi,
$$

where $\left\{e_{1}, \ldots, e_{n}\right\}$ is a local orthonormal frame of $T \Sigma$ and $H=\operatorname{tr} A$ is the mean curvature of $\Sigma$ corresponding to the orientation $N$. Using (13) and (11), it is straightforward to see that the skew-commutativity rule

$$
\not D(\gamma(N) \psi)=-\gamma(N) \not D \psi
$$

holds for any spinor field $\psi \in \Gamma(\$ \Sigma)$. It is important to point out that, from this fact, the spectrum of $\not D$ is always symmetric with respect to zero, while this is the case for the Dirac operator $D_{\Sigma}$ of the intrinsic spinor bundle only when $n$ is even. Indeed, in this case, we have an isomorphism of Dirac bundles

$$
(\$ \Phi, \gamma, \not D) \equiv\left(\mathbb{S} \Sigma, \gamma_{\Sigma}, D_{\Sigma}\right),
$$

and the decomposition $\$ \Phi=\$ \Sigma^{+} \oplus \$ \Sigma^{-}$, given by

$$
\$ \Sigma^{ \pm}:=\{\psi \in \$ \Sigma \mid i \gamma(N) \psi= \pm \psi\},
$$

corresponds to the chiral decomposition of the spinor bundle $\mathbb{S} \Sigma$. Hence $\not D$ interchanges $\$ \Sigma^{+}$and $\$ \Sigma^{-}$.

When $n$ is odd the spectrum of $D_{\Sigma}$ is not necessarily symmetric. In fact, in this case, the spectrum of $\not D$ is just the symmetrization of the spectrum of $D_{\Sigma}$. 
This is why the decomposition of $\mathbb{S} M$ into positive and negative chiral spinors induces an orthogonal and $\gamma, \not D$-invariant decomposition $\$ \Sigma=\$ \Sigma_{+} \oplus \$ \Sigma_{-}$, with $\$ \Sigma_{ \pm}:=\left(\mathbb{S} M^{ \pm}\right)_{\mid \Sigma}$, in such a way that

$$
\left(\$ \Sigma_{ \pm}, \gamma, \not D_{\mid \$ \Sigma_{ \pm}}\right) \equiv\left(\mathbb{S} \Sigma, \pm \gamma_{\Sigma}, \pm D_{\Sigma}\right) \text {. }
$$

Also, $\gamma(N)$ interchanges the decomposition, and both maps $\gamma(N): \$ \Sigma_{ \pm} \rightarrow \$ \Sigma_{\mp}$ are isomorphisms.

Consequently, studying the spectrum of the induced operator $\not D$ is equivalent to studying the spectrum of the Dirac operator $D_{\Sigma}$ of the Riemannian spin structure induced on the hypersurface $\Sigma$.

2.3. A spinorial Reilly-type inequality for manifolds with boundary. Here, we prove a spinorial Reilly-type inequality (see [Liu and Yau 2003] and [Raulot 2013]).

Recall that on a compact $(n+1)$-dimensional Riemannian spin manifold $M$ with boundary $\Sigma=\partial M$, for any spinor field $\psi \in \Gamma(\mathbb{S} M)$, the fundamental SchrödingerLichnerowicz formula is given by:

$$
\int_{\Sigma}\left(\langle\not D \psi, \psi\rangle-\frac{H}{2}|\psi|^{2}\right) d \Sigma=\int_{M}\left(\frac{1}{4} R|\psi|^{2}+|\nabla \psi|^{2}-|D \psi|^{2}\right) d M,
$$

where $R$ is the scalar curvature of $M$. Note that the assumption $R \geq 0$ is quite natural and has been used intensively to get, in particular, lower bounds on both $D$ and $\not D$. However, in our situation (see Section 3.1), we have a weaker assumption on the scalar curvature. More precisely, we assume that there exists a smooth vector field $X \in \Gamma(T M)$ such that

$$
R \geq 2|X|^{2}+2 \delta(X),
$$
where $|X|^{2}=g(X, X)$ and $\delta$ is the divergence of $X=\sum_{j=1}^{n} X^{j} e_{j} \in \Gamma(T M)$, locally
given by

$$
\delta(X)=-\sum_{i=1}^{n+1} e_{i}\left(X^{i}\right)
$$

Then we prove an adapted Reilly-type inequality. Namely:

Proposition 6. Let $M$ a compact Riemannian spin manifold with boundary $\Sigma$ such that there exists a smooth vector field $X \in \Gamma(T M)$ satisfying (16). Then

$$
\int_{\Sigma}\left\langle\not D \psi-\frac{1}{2}(H+g(X, N)) \psi, \psi\right\rangle d \Sigma \geq \int_{M}\left(\frac{1}{2}|\nabla \psi|^{2}-|D \psi|^{2}\right) d M .
$$

Moreover, equality occurs if and only if the spinor field $\psi$ satisfies

$$
\nabla_{Y} \psi=-g(X, Y) \psi
$$

for all $Y \in \Gamma(T M)$. 
Proof. First note that, since

$$
\delta\left(|\psi|^{2} X\right)=-X\left(|\psi|^{2}\right)+|\psi|^{2} \delta(X),
$$

the Stokes formula gives

$$
\begin{aligned}
\int_{M} \frac{R}{4}|\psi|^{2} d M= & \int_{M}\left(\frac{R}{4}-\frac{1}{2} \delta(X)\right)|\psi|^{2} d M+\frac{1}{2} \int_{M} \delta(X)|\psi|^{2} d M \\
= & \frac{1}{4} \int_{M}(R-2 \delta(X))|\psi|^{2} d M+\frac{1}{2} \int_{M} X\left(|\psi|^{2}\right) d M \\
& +\frac{1}{2} \int_{\Sigma} g(X, N)|\psi|^{2} d \Sigma .
\end{aligned}
$$

Inserting this identity in (15) leads to

$$
\begin{aligned}
\int_{\Sigma}\langle\not D \psi & \left.-\frac{1}{2}(H+g(X, N)), \psi\right\rangle d \Sigma \\
= & \int_{M}\left(\frac{1}{4}(R-2 \delta(X))|\psi|^{2}+\frac{1}{2} X\left(|\psi|^{2}\right)\right) d M+\int_{M}\left(|\nabla \psi|^{2}-|D \psi|^{2}\right) d M
\end{aligned}
$$

and, using (16), we conclude that

$$
\begin{aligned}
\int_{\Sigma}\langle\not D \psi & \left.-\frac{1}{2}(H+g(X, N)), \psi\right\rangle d \Sigma \\
& \geq \int_{M}\left(\frac{1}{2}|X|^{2}|\psi|^{2}+\frac{1}{2} X\left(|\psi|^{2}\right)\right) d M+\int_{M}\left(|\nabla \psi|^{2}-|D \psi|^{2}\right) d M .
\end{aligned}
$$

If we let $\widetilde{\nabla}_{Y} \psi:=\nabla_{Y} \psi+g(X, Y) \psi$, it is straightforward to compute

$$
|\widetilde{\nabla} \psi|^{2}=|\nabla \psi|^{2}+|X|^{2}|\psi|^{2}+2 \operatorname{Re}\left\langle\nabla_{X} \psi, \psi\right\rangle,
$$

and since $2 \operatorname{Re}\left\langle\nabla_{X} \psi, \psi\right\rangle=X\left(|\psi|^{2}\right)$, we get

$$
\frac{1}{2} X\left(|\psi|^{2}\right) \geq-\frac{1}{2}|\nabla \psi|^{2}-\frac{1}{2}|X|^{2}|\psi|^{2},
$$

with equality if and only if $\widetilde{\nabla} \psi=0$. Combining this last inequality with (19) finishes the proof.

2.4. A local boundary elliptic condition for the Dirac operator. As before, $\Sigma$ is the boundary of an $(n+1)$-dimensional Riemannian spin compact manifold $M$. We define two pointwise projections

$$
P_{ \pm}: \$ \Sigma \longrightarrow \$ \Sigma
$$

on the induced Dirac bundle over the hypersurface by

$$
P_{ \pm}=\frac{1}{2}\left(\operatorname{Id}_{\$ \Sigma} \pm i \gamma(N)\right) .
$$


It is a well-known fact that these two orthogonal projections $P_{ \pm}$acting on the spin bundle $\$ \Sigma$ provide local elliptic boundary conditions for the Dirac operator $D$ of $M$. The ellipticity of these boundary conditions and that of the Dirac operator $D$ allow us to solve boundary value problems for $D$ on $M$ by prescribing, on the boundary $\Sigma$, the corresponding $P_{ \pm}$-projections of the solutions. Namely, we have:

Proposition 7 [Hijazi and Montiel 2014]. Let $M$ be a compact Riemannian spin manifold with boundary a hypersurface $\Sigma$. If $\varphi \in \Gamma(\$ \Sigma)$ is a smooth spinor field of the induced Dirac bundle, then the boundary value problem

$$
\begin{cases}D \psi=0 & \text { on } M \\ P_{ \pm}\left(\psi_{\mid \Sigma}\right)=P_{ \pm} \varphi & \text { on } \Sigma\end{cases}
$$

for the Dirac operator has a unique smooth solution $\psi \in \Gamma(\mathbb{S} M)$.

For a more general discussion on boundary conditions for the Dirac operator, we refer to [Booß-Bavnbek and Wojciechowski 1993], [Ballmann and Bär 2012] or [Bartnik and Chruściel 2005].

2.5. A holographic principle for the existence of parallel spinors. It is by now standard (see [Hijazi et al. 2001b; 2002]) to make use of (15) for a compact Riemannian spin manifold $M$ with nonnegative scalar curvature $R$, together with the solution of an appropriate boundary value problem for the Dirac operator $D$ of $M$, in order to establish a certain integral inequality for the induced Dirac operator $\not D$ of the boundary hypersurface $\partial M=\Sigma$. Raulot [2013] uses such arguments for compact manifolds whose scalar curvature satisfies (16). In this section, we generalize the holographic principle for the existence of parallel spinors proved in [Hijazi and Montiel 2014] in the context studied in [Raulot 2013].

First we need to recall the following fact:

Lemma 8 [Hijazi et al. 2002]. For any smooth spinor field $\psi \in \Gamma(\$ \$)$,

$$
\int_{\Sigma}\langle\not D \psi, \psi\rangle d \Sigma=2 \int_{\Sigma}\left\langle\not D P_{+} \psi, P_{-} \psi\right\rangle d \Sigma .
$$

The proof simply relies on the self-adjointness of the Dirac operator $\not D$ and on the identities

$$
\not D P_{ \pm}=P_{\mp} \not D,
$$

which are obtained using (14) and (20).

Proposition 9. Let $M$ be a compact Riemannian spin manifold with scalar curvature satisfying (16) such that

$$
F:=H+g(X, N)>0 .
$$


For any $\varphi \in \Gamma(\$ \Sigma)$, one has

$$
0 \leq \int_{\Sigma}\left(\frac{1}{F}\left|\not D P_{+} \varphi\right|^{2}-\frac{F}{4}\left|P_{+} \varphi\right|^{2}\right) d \Sigma .
$$

Moreover equality holds if and only if there exists a parallel spinor field $\psi \in \Gamma(S M)$ such that $P_{+} \psi=P_{+} \varphi$ along the boundary hypersurface $\Sigma$ and the vector field $X$ vanishes identically on $M$.

Proof. Take any spinor field $\varphi \in \Gamma(\$ \Phi \Sigma)$ of the induced spinor bundle on the hypersurface and consider the boundary value problem

$$
\begin{cases}D \psi=0 & \text { on } M \\ P_{+} \psi=P_{+} \varphi & \text { on } \Sigma\end{cases}
$$

for the Dirac operator $D$ and the boundary condition $P_{+}$. The existence and uniqueness of a smooth solution $\psi \in \Gamma(\mathbb{S} M)$ for this boundary problem is ensured by Proposition 7 . This solution $\psi$, inserted in inequality (17), translates to

$$
0 \leq \frac{1}{2} \int_{M}|\nabla \psi|^{2} d M \leq \int_{\Sigma}\left(\langle\not D \psi, \psi\rangle-\frac{F}{2}|\psi|^{2}\right) d \Sigma .
$$

Note that if equality is achieved, then $\psi$ is a parallel spinor field satisfying (18). Since such a spinor field has no zeros, the vector field $X$ vanishes identically on the whole of $M$. Inequality (23) combined with Lemma 8, together with the fact that the decomposition

$$
\psi=P_{+} \psi+P_{-} \psi
$$

is pointwise orthogonal, imply

$$
0 \leq \int_{\Sigma}\left(2\left\langle\not D P_{+} \psi, P_{-} \psi\right\rangle-\frac{F}{2}\left|P_{+} \psi\right|^{2}-\frac{F}{2}\left|P_{-} \psi\right|^{2}\right) d \Sigma .
$$

Since the function $F$ is assumed to be positive on $\Sigma$, it follows that

$0 \leq\left|\sqrt{\frac{2}{F}} \not D P_{+} \psi-\sqrt{\frac{F}{2}} P_{-} \psi\right|^{2}=\frac{2}{F}\left|\not D P_{+} \psi\right|^{2}+\frac{F}{2}\left|P_{-} \psi\right|^{2}-2\left\langle\not D P_{+} \psi, P_{-} \psi\right\rangle$.

In other words,

$$
2\left\langle\not D P_{+} \psi, P_{-} \psi\right\rangle-\frac{F}{2}\left|P_{-} \psi\right|^{2} \leq \frac{2}{F}\left|\not D P_{+} \psi\right|^{2},
$$

which, when combined with inequality (24), implies inequality (22). Now, if equality holds, we already noticed that the spinor field $\psi$ must be parallel with $P_{+} \psi=P_{+} \varphi$ and $X \equiv 0$.

Conversely, if we assume that there is a parallel spinor field $\psi$ on $M$ and $X \equiv 0$, then we are in the situation covered in [Hijazi and Montiel 2014]. 
With this, we are ready to state the main result of this section:

Theorem 10. Let $M$ be a compact Riemannian spin $(n+1)$-dimensional manifold, and $X \in \Gamma(T M)$ such that

$$
R \geq 2|X|^{2}+2 \delta(X) \text { and } F:=H+g(X, N)>0 .
$$

Then, for any spinor field $\varphi \in \Gamma(\$ \Sigma)$, one has

$$
0 \leq \int_{\Sigma}\left(\frac{1}{F}|\not D \varphi|^{2}-\frac{F}{4}|\varphi|^{2}\right) d \Sigma .
$$

Equality holds if and only if there exist two parallel spinor fields $\Psi^{+}, \Psi^{-} \in \Gamma(\mathbb{S} M)$ such that $P_{+} \Psi^{+}=P_{+} \varphi$ and $P_{-} \Psi^{-}=P_{-} \varphi$ on the boundary and $X \equiv 0$.

Proof. From the symmetry between the two boundary conditions $P_{+}$and $P_{-}$for the Dirac operator on $M$ (see Proposition 7 and Lemma 8), one can repeat the proof of Proposition 9 to get the inequality corresponding to (22) where the positive projection $P_{+}$is replaced by the negative one $P_{-}$. Hence, for any spinor field $\varphi \in \Gamma(\$ \Phi)$, we also have

$$
0 \leq \int_{\Sigma}\left(\frac{1}{F}\left|\not D P_{-} \varphi\right|^{2}-\frac{F}{4}\left|P_{-} \varphi\right|^{2}\right) d \Sigma .
$$

Taking into account the relation (21) and the pointwise orthogonality of the projections $P_{ \pm}$, the sum of the two inequalities (22) and (26) yields (25). The equality case is a consequence of Proposition 9.

Remark 11. Note that, as observed in [Hijazi and Montiel 2014], equality in (25) does not imply that the two parallel spinors in Theorem 10 coincide.

We should also mention that inequality (25) has a nice interpretation in terms of the first eigenvalue of the boundary Dirac operator $\not D_{F}$ associated with the conformal metric $g_{F}=F^{2} g$. More precisely:

Corollary 12. Let $\left(M^{n+1}, g\right)$ be an $(n+1)$-dimensional compact connected Riemannian spin manifold satisfying the assumptions of Theorem 10. Then the first nonnegative eigenvalue $\lambda_{1}\left(\not D_{F}\right)$ of the Dirac operator corresponding to the conformal metric $g_{F}=F^{2} g$ satisfies

$$
\lambda_{1}\left(\not D_{F}\right) \geq \frac{1}{2}
$$

and equality holds if and only if $M$ admits a nontrivial parallel spinor (and $X \equiv 0)$. In this case, the eigenspace corresponding to $\lambda_{1}\left(\not D_{F}\right)=\frac{1}{2}$ consists of restrictions to $\Sigma$ of parallel spinor fields on $M$ multiplied by the function $F^{-(n-1) / 2}$. Furthermore the boundary hypersurface $\Sigma$ has to be connected.

The proof is omitted since it is similar to [Hijazi and Montiel 2014, Theorem 1]. 
2.6. A discussion on quasilocal masses. In this section, we consider a 3-dimensional compact connected Riemannian manifold $\left(M^{3}, g\right)$ with nonnegative scalar curvature, whose boundary $\Sigma^{2}$ has positive mean curvature $H$. Note that since $M$ is a 3-dimensional manifold, it is necessarily spin. Moreover we also assume that there exists an immersion $\iota_{0}$ of the surface $\Sigma$ in $\mathbb{R}^{3}$ with mean curvature $H_{0}$.

One of the fundamental results in classical general relativity is certainly the proof of the positivity of the total energy by Schoen and Yau [1981] and Witten [1981]. This led to the more ambitious claim of associating energy to extended, but finite, spacetime domains, that is, at the quasilocal level. Obviously the quasilocal data could provide a more detailed characterization of the states of the gravitational field than the global ones, so they are interesting in their own right. For a complete review of these topics, we refer to [Szabados 2004]. It is currently required that a quasilocal mass satisfies natural properties, among which are:

(I) Nonnegativity: $\mu(\Sigma) \geq 0$.

(II) Rigidity: $\mathcal{M}(\Sigma)=0$ if and only if $\Sigma$ is in the Minkowski spacetime.

(III) Monotonicity: If $\Sigma_{1}=\partial M_{1}$ and $\Sigma_{2}=\partial M_{2}$ such that $M_{1} \subset M_{2}$, then $M\left(\Sigma_{1}\right) \leq M\left(\Sigma_{2}\right)$.

(IV) ADM limit: If $\left(\Sigma_{k}\right)$ is a sequence of surfaces that exhaust an asymptotically flat manifold $\left(N^{3}, g\right)$, then

$$
\lim _{k \rightarrow \infty} M\left(\Sigma_{k}\right)=m_{\mathrm{ADM}}(g),
$$

where $m_{\mathrm{ADM}}(g)$ is the $\mathrm{ADM}$ mass of $(N, g)$.

(V) Black hole limit: If $\Sigma$ is a horizon in an asymptotically flat manifold $\left(N^{3}, g\right)$, then

$$
\mu(\Sigma)=\sqrt{\frac{A}{16 \pi}},
$$

where $A$ is the area of $\Sigma$.

Brown and York [1993] proposed the following definition for the quasilocal mass of a surface $\Sigma$ (now called the Brown-York mass):

$$
m_{B Y}(\Sigma):=\frac{1}{8 \pi} \int_{\Sigma}\left(H_{0}-H\right) d \Sigma .
$$

The nonnegativity of $m_{B Y}(\Sigma)$ is proved in [Shi and Tam 2002] under additional assumptions. Indeed they impose that $\iota_{0}$ is a strictly convex isometric embedding, which by the Weyl embedding theorem [1916] is equivalent to the fact that $\Sigma$ has positive Gauss curvature. Moreover, in this situation, the embedding $\iota_{0}$ is unique up to an isometry of $\mathbb{R}^{3}$. 
Recently Lam [2011] proposed in his thesis the definition

$$
m_{L}(\Sigma):=\frac{1}{16 \pi} \int_{\Sigma} \frac{1}{H_{0}}\left(H_{0}^{2}-H^{2}\right) d \Sigma .
$$

He proves that $m_{L}(\Sigma)$ has several interesting properties for certain surfaces in complete asymptotically flat Riemannian manifolds that are the graphs of smooth functions over $\mathbb{R}^{3}$ (see the same work for a precise description). More precisely, it satisfies Properties (I), (III), (IV) and (V). Moreover, using the Cauchy-Schwarz inequality, it is straightforward to check that $m_{B Y}(\Sigma) \geq m_{L}(\Sigma)$.

From [Hijazi and Montiel 2014], we can define a quasilocal mass similar to the Brown-York and Lam masses, and prove its nonnegativity in the more general context described in the beginning of this section. Indeed, if we let

$$
m(\Sigma):=\frac{1}{16 \pi} \int_{\Sigma} \frac{1}{H}\left(H_{0}^{2}-H^{2}\right) d \Sigma,
$$

then, from the immersion $\iota_{0}$, there exists a spinor field $\Psi_{0} \in \Gamma(\$ \Sigma)$ satisfying the Dirac equation

$$
\not D \Psi_{0}=\frac{H_{0}}{2} \Psi_{0} \quad \text { and } \quad\left|\Psi_{0}\right|=1 .
$$

It is obtained by taking the restriction to $\Sigma$ of a parallel spinor field on $\mathbb{R}^{3}$. Now taking $\Psi_{0}$ in inequality (25) with $X \equiv 0$ and $F=H$ gives $m(\Sigma) \geq 0$. Moreover, from the same reference, $m(\Sigma)=0$ if and only if $M$ is a Euclidean domain and the embedding of $\Sigma$ in $M$ and its immersion in $\mathbb{R}^{3}$ are congruent. In other words, properties (I) and (II) are satisfied.

Note that if we assume that $\Sigma$ has positive Gauss curvature (which is a stronger assumption) then using the Cauchy-Schwarz inequality implies that $m(\Sigma) \geq m_{B Y}(\Sigma)$, and the nonnegativity of $m(\Sigma)$ follows from the nonnegativity of the Brown-York mass. On the other hand, it is also proved in [Hijazi and Montiel 2014, Proof of Corollary 10] that (IV) holds. However it is clear from the definition that the mass $m(\Sigma)$ is not defined for minimal surfaces (and so for apparent horizons). Moreover the monotonicity property (III) is not satisfied in general. Take for example the 3-dimensional Schwarzschild manifold $\left(N^{3}, g\right)=\left(\mathbb{R}^{3} \backslash\{0\}, u^{4} g_{\text {eucl }}\right)$, where $u:=1+M / 2 r, M>0$, and $g_{\text {eucl }}$ is the Euclidean metric. For a sphere $\mathbb{S}_{r}^{2}$ in $N^{3}$, its isometric image in $\mathbb{R}^{3}$ is $\mathbb{S}_{r u^{2}}^{2}$. Thus $H_{0}=2 / r u^{2}$ and since the Schwarzschild metric is conformal to the Euclidean metric,

$$
H=u^{-2}\left(\frac{2}{r}+\frac{4}{u} \frac{\partial u}{\partial r}\right)
$$


A direct computation gives

$$
m\left(\mathbb{S}_{r}^{2}\right)=M \frac{r+M / 2}{r-M / 2},
$$

and so $m\left(\mathbb{S}_{r}^{2}\right)$ is monotonically decreasing to the ADM mass $M$ as $r$ goes to infinity.

\section{Spacelike surfaces in initial data sets}

3.1. The Jang equation. In this section, we recall some well-known facts about the Jang equation (for more details, we refer to [Schoen and Yau 1981], [Yau 2001] or [Andersson et al. 2011]). This equation first was used by Jang [1978] in his attempt to prove the positive mass theorem using the inverse mean curvature flow. However, as shown by Schoen and Yau [1981], this equation can be used to reduce the proof of the general positive mass theorem to the case of time-symmetric initial data sets (that is, $K_{i j}=0$ ) previously obtained by the same authors [1979]. More recently, Liu and Yau [2003; 2006] defined a quasilocal mass, generalizing the Brown-York quasilocal mass, and proved its positivity using the Jang equation. Other similar applications of the Jang equation can be found in, for example, [Wang and Yau 2007; 2009].

The problem can be stated as follows: Let $\left(M^{3}, g, K\right)$ be an initial data set for the Einstein equation and consider the four-dimensional manifold $M \times \mathbb{R}$ equipped with the Riemannian metric $\langle\cdot, \cdot\rangle:=g \oplus d t^{2}$. The problem is to find a smooth function $u: M \rightarrow \mathbb{R}$ such that the hypersurface $\widehat{M}$ of $M \times \mathbb{R}$ obtained by taking the graph of $u$ over $M$ satisfies the equation

$$
H_{\widehat{M}}=\operatorname{tr}_{\widehat{M}}(K),
$$

where $H_{\widehat{M}}$ denotes the mean curvature of $\hat{M}$ in $(M \times \mathbb{R},\langle\cdot, \cdot\rangle)$ and $\operatorname{tr}_{\widehat{M}}(\cdot)$ is the trace on $\widehat{M}$ with respect to the induced metric. This geometric problem is equivalent to solving the nonlinear second-order elliptic equation

$$
\sum_{i, j=1}^{3}\left(g^{i j}-\frac{u^{i} u^{j}}{1+|\nabla u|^{2}}\right)\left(\frac{\left(\nabla^{2} u\right)_{i j}}{\sqrt{1+|\nabla u|^{2}}}-K_{i j}\right)=0
$$

where $\nabla$ (respectively, $\nabla^{2}$ ) denotes the Levi-Civita connection (respectively, the Hessian) of the metric $g, u^{i}=g^{i j} u_{j}$ and $u_{j}=e_{j}(u)$. Note that the metric induced by $\langle\cdot, \cdot\rangle$ on $\widehat{M}$ is

$$
\hat{g}_{i j}=g_{i j}+u_{i} u_{j}
$$

and can be viewed as a deformation of the metric $g$ on $M$. In the following, we adopt the convention that $M$ and $\widehat{M}$ denote, respectively, the Riemannian 
manifolds $(M, g)$ and $(M, \hat{g})$. Analogously, if $\nabla$ denotes the Levi-Civita connection for $M$, then $\hat{\nabla}$ denotes that on $\widehat{M}$ and so on. Since we assume that the initial data set $\left(M^{3}, g, K\right)$ comes from a spacetime satisfying the dominant energy condition, we have that the relation

$$
0 \leq 2(\mu-|J|) \leq \widehat{R}-2|X|_{\hat{g}}^{2}-2 \widehat{\delta}(X)
$$

holds on $\widehat{M}$, where

$$
X=\omega-\hat{\nabla} \log (f),
$$

$\omega$ is the tangent part of the vector field dual to $-K(\cdot, \hat{v}), f=-\left\langle\partial_{t}, \widehat{v}\right\rangle$ and $\hat{v}$ denotes the unit normal vector field to $\hat{M}$ in $M \times \mathbb{R}$. All the quantities $K_{i j}, \mu$ and $J$ are defined on $M \times \mathbb{R}$ by parallel transport along the $\mathbb{R}$-factor. Moreover equality occurs in (28) if and only if $\mu=|J|$ and the second fundamental form of $\hat{M}$ in $M \times \mathbb{R}$ is $K$.

It is important to note here that in Theorem 2 we assume that there is no apparent horizon in the interior of $\Omega$ so that there exists a global solution of the Jang equation which does not blow up.

3.2. Proof of Theorem 2. From [Yau 2001], and since we assumed that $\Omega$ has no apparent horizon in its interior, there exists a smooth solution $u$ on $\Omega$ of the Jang equation (27), defined with the Dirichlet boundary condition

$$
u_{\mid \Sigma} \equiv 0
$$

This boundary condition ensures that the metrics $\hat{g}$ and $g$ coincide on the boundary $\Sigma$ so that the Dirac operators $\not D$ acting on $\$ \Phi$ and $\widehat{D}$ on $\widehat{\Phi} \Sigma$ also coincide. Moreover, from a calculation in the same work,

$$
\hat{H}-\hat{g}(X, \hat{N})=f^{-1} H-\sigma|\nabla u| \operatorname{tr}_{\Sigma}(K),
$$

where $\hat{N}$ denotes the unit outward normal vector field of $\Sigma$ in $\widehat{\Omega}$ and $\sigma \in\{ \pm 1\}$. From this equality and since $f=-\left\langle\partial_{t}, \widehat{v}\right\rangle=1 / \sqrt{1+|\nabla u|^{2}}$, we easily see that

$$
F:=\hat{H}-\hat{g}(X, \hat{N}) \geq|\mathscr{H}|=\sqrt{H^{2}-\operatorname{tr}_{\Sigma}(K)^{2}} .
$$

Since we assume that $\Sigma$ has a spacelike mean curvature vector $\mathscr{H}$, this implies that the function $F$ is positive on $\Sigma$. From the discussion of Section 3.1, we also have that the resulting Riemannian manifold $\widehat{\Omega}$ satisfies the condition (16) because of (28), the vector field $X$ being defined here by (29). Clearly all the assumptions of Theorem 10 are fulfilled and we deduce that for all $\varphi \in \Gamma(\$ \Sigma)$,

$$
0 \leq \int_{\Sigma}\left(\frac{1}{F}|\not D \varphi|^{2}-\frac{F}{4}|\varphi|^{2}\right) d \Sigma
$$


which by inequality (30) implies inequality (2).

Now assume that equality is achieved. Once again we apply Theorem 10, and then $\widehat{\Omega}$ has at least a parallel spinor field $\Phi$. In particular, $\widehat{\Omega}$ is Ricci-flat, and since it is a 3-dimensional domain, it is flat. Moreover, if we have equality in (28), then the second fundamental form of $\widehat{\Omega}$ in $M \times \mathbb{R}$ is $K_{i j}$. So we can choose a coordinate system $\hat{x}=\left(\hat{x}_{1}, \hat{x}_{2}, \hat{x}_{3}\right)$ in a neighborhood $U$ of a point $p \in \Omega$ such that $\hat{g}_{i j}=\delta_{i j}$. In this chart,

$$
g_{i j}=\delta_{i j}-\frac{\partial u}{\partial \hat{x}_{i}} \frac{\partial u}{\partial \hat{x}_{j}},
$$

and this shows that if $\left(\hat{x}_{1}, \widehat{x_{2}}, \widehat{x_{3}}, t\right)$ denotes coordinates in the Minkowski spacetime, the graph of $u$ over $U$ isometrically embeds in $\mathbb{R}^{3,1}$ with second fundamental form given by $K_{i j}$. Then it is clear that $\Omega$ locally embeds in the Minkowski spacetime with $K$ as second fundamental form as asserted.

As a first consequence, we have the estimate proved by Raulot [2013] for the first eigenvalue of the Dirac operator on $\Sigma$.

Corollary 13. Under the same conditions of Theorem 2, the first eigenvalue $\lambda_{1}\left(D_{\Sigma}\right)$ of the Dirac operator satisfies

$$
\lambda_{1}\left(D_{\Sigma}\right)^{2} \geq \frac{1}{4} \inf _{\Sigma}|\mathscr{H}|^{2} .
$$

Moreover, if equality occurs, then $\Sigma$ is connected and there exists a local isometric embedding of $\Omega$ as a spacelike hypersurface in $\mathbb{R}^{3,1}$ with $K$ as second fundamental form.

Proof. The inequality on $\lambda_{1}\left(D_{\Sigma}\right)$ follows directly by taking $\varphi=\Phi \in \Gamma(\$ \Sigma)$ in (2), where $\Phi$ is an eigenspinor for the Dirac operator $\not D$ associated with the eigenvalue $\lambda_{1}(\not D)$ (which equals $\lambda_{1}\left(D_{\Sigma}\right)$ ). On the other hand, the second part of the equality case follows directly from Theorem 2 . For the connectedness of $\Sigma$, it is enough to remark that, from [Hijazi et al. 2001a], the eigenspace associated to $\lambda_{1}(\not D)$ corresponds to the restriction to $\Sigma$ of the space of parallel spinor fields on the domain $\widehat{\Omega}$ obtained by solving the Jang equation. Then, assuming that $\Sigma$ has several connected components, we fix one of them, say $\Sigma_{0}$, and define a spinor field on $\Sigma$ by

$$
\widetilde{\Phi}= \begin{cases}\Phi_{0} & \text { on } \Sigma_{0} \\ 0 & \text { on } \Sigma-\Sigma_{0}\end{cases}
$$

where $\Phi_{0}$ is an eigenspinor for the extrinsic Dirac operator $\not D$ associated to the eigenvalue $\lambda_{1}(\not D)$. It is then straightforward to check that $\widetilde{\Phi}$ is also an eigenspinor associated to $\lambda_{1}(\not D)$ so that it comes from the restriction of a parallel spinor on $\hat{\Omega}$. However, since such a spinor field has constant norm, it is impossible unless $\Sigma$ is connected. 
Proof of Theorem 3. In order to establish inequality (3) it is sufficient to apply inequality (2) to the restriction to $\Sigma$ of a parallel spinor field on $\mathbb{R}^{3}$. From the equality case of Theorem 2, we deduce that $\Omega$ locally embeds in the Minkowski spacetime with $K$ as a second fundamental form. On the other hand, we have equality in (30) so that $\widehat{H}=|\mathscr{H}|$, and then equality in (3) now reads

$$
\int_{\Sigma}\left(\hat{H}-\frac{H_{0}^{2}}{\hat{H}}\right) d \Sigma=0 \text {. }
$$

We conclude by applying the rigidity part of [Hijazi and Montiel 2014, Theorem 3] to the compact Ricci-flat manifold $\widehat{\Omega}$ to deduce that $\Sigma$ is connected and $|\mathscr{H}|=H_{0}$.

\subsection{Codimension-two outer untrapped submanifolds in the Minkowski space-}

time. In this section, we prove that inequality (2) holds in the case of codimensiontwo outer untrapped submanifolds of the Minkowski spacetime without any assumption on the existence of apparent horizon. More precisely, we prove:

Theorem 14. Let $\Sigma^{n}$ be a codimension-two outer untrapped submanifold of the $(n+2)$-dimensional Minkowski spacetime $\left(\mathbb{R}^{n+1,1},\langle\cdot, \cdot\rangle\right)$. Then inequality (2) holds. Moreover equality holds if and only if $\Sigma$ lies in a hyperplane of $\mathbb{R}^{n+1,1}$.

Proof. First we note that by assumption $\Sigma$ factorizes through a compact and connected spacelike hypersurface $\Omega$ of $\mathbb{R}^{n+1,1}$. This factorization provides us a Lorentzian orthonormal reference $\{T, N\}$ for the normal plane of $\Sigma$ in $\mathbb{R}^{n+1,1}$, and, since $\Sigma$ is the boundary of a mean-convex domain $\Omega$ and has spacelike mean curvature vector, we deduce that the corresponding future-directed null expansions satisfy $\theta_{+}>0$ and $\theta_{-}<0$. On the other hand, from the work of Bartnik and Simon [1982] and a straightforward generalization in [Miao et al. 2010, Lemma 4.1], the submanifold $\Sigma$ spans a compact, smoothly immersed, maximal hypersurface $\Omega^{\prime}$ in $\mathbb{R}^{n+1,1}$. This means that $\Sigma$ factorizes through another spacelike hypersurface $\Omega^{\prime}$ of $\mathbb{R}^{n+1,1}$. The new factorization provides us a different Lorentzian orthonormal reference $\left\{T^{\prime}, N^{\prime}\right\}$ for the normal plane of $\Sigma$ in $\mathbb{R}^{n+1,1}$. In fact, it is obvious that there must be a function $f \in C^{\infty}(\Sigma)$ such that

$$
T^{\prime}=(\cosh f) T-(\sinh f) N \quad \text { and } \quad N^{\prime}=-(\sinh f) T+(\cosh f) N .
$$

It is clear that this new reference determines a new pair of null vectors $T^{\prime} \pm N^{\prime}$ and a new future-directed null expansion of $\mathscr{H}$

$$
\theta_{+}^{\prime}=e^{f} \theta_{+} \quad \text { and } \quad \theta_{-}^{\prime}=e^{-f} \theta_{-}
$$

which satisfies $\theta_{+}^{\prime}>0$ and $\theta_{-}^{\prime}<0$. In particular, we get that $2 H^{\prime}=\theta_{+}^{\prime}-\theta_{-}^{\prime}>0$. Moreover, since $\Omega^{\prime}$ is maximal, we have $\operatorname{tr}\left(K^{\prime}\right)=0$, and the Gauss formula gives $R^{\prime}=\left|K^{\prime}\right|^{2} \geq 0$. Here $R^{\prime}$ is the scalar curvature of $\Omega^{\prime}$ equipped with the metric 
induced by the Minkowski spacetime, and $K^{\prime}$ is the associated second fundamental form. On the other hand, since $\Sigma$ has a spacelike mean curvature vector, we deduce

$$
0<|\mathscr{H}|=\sqrt{-\theta_{+}^{\prime} \theta_{-}^{\prime}}=\sqrt{H^{\prime 2}-\operatorname{tr}_{\Sigma}\left(K^{\prime}\right)^{2}} \leq H^{\prime},
$$

so we conclude that $\Omega^{\prime}$ is such that $R^{\prime} \geq 0$ and $H^{\prime}>0$. Now we can apply Theorem 10 to $\Omega^{\prime}$ with $X \equiv 0$, and then for all $\varphi \in \Gamma(\$ \Phi)$,

$$
0 \leq \int_{\Sigma}\left(\frac{1}{H^{\prime}}|\not D \varphi|^{2}-\frac{1}{4} H^{\prime}|\varphi|^{2}\right) d \Sigma .
$$

Inequality (2) follows using inequality (32). Assume now that equality is achieved. From the equality case of (33), we deduce that $\Omega^{\prime}$ has at least a parallel spinor so that $\Omega^{\prime}$ is Ricci-flat. In particular, it has zero scalar curvature, and since $R^{\prime}=\left|K^{\prime}\right|^{2}=0$, $\Omega^{\prime}$ has to be totally geodesic in $\mathbb{R}^{n+1,1}$, hence $\Sigma$ lies in a hyperplane of $\mathbb{R}^{n+1,1}$. Conversely, if $\Sigma$ is a codimension-two submanifold with spacelike mean curvature vector which lies in a hyperplane $\mathbb{R}^{n+1,1}$, then its second fundamental form $K$ is zero since a hyperplane $P^{n+1}$ is totally geodesic. In particular, the squared norm of the mean curvature vector of $\Sigma$ satisfies

$$
|\mathscr{H}|^{2}=H^{2}-\operatorname{tr}_{\Sigma}(K)^{2}=H^{2},
$$

where $H$ is the mean curvature of $\Sigma$ in the hyperplane $P$. Note that $|\mathscr{H}|>0$ since $H>0$. Consider now a parallel spinor field $\Phi_{0}$ on $\mathbb{R}^{n+1,1}$. The spinorial Gauss formula from the totally geodesic immersion of the hyperplane $P^{n+1}$ in $\mathbb{R}^{n+1,1}$ and then the one from $\Sigma^{n}$ into $P^{n+1}$ tell us that $\Phi_{0}$ satisfies

$$
\not_{Y} \Phi_{0}=-\frac{1}{2} \gamma(A Y) \Phi_{0}
$$

for all $Y \in \Gamma(T \Sigma)$, where $A$ is the Weingarten map of $\Sigma^{n}$ in $P^{n+1}$. Taking the trace of this identity gives

$$
\not D \Phi_{0}=\frac{1}{2} H \Phi_{0}=\frac{1}{2}|\mathscr{H}| \Phi_{0},
$$

where the last equality comes from (34). It is now straightforward to check that equality holds in (2) for $\varphi=\Phi_{0}$.

Note that Theorem 4 is obtained as a direct application of the previous result. As an application we obtain the $n$-dimensional counterpart of Corollary 13 in the Minkowski spacetime with an optimal rigidity statement:

Corollary 15. Let $\Sigma^{n}$ be a codimension-two outer untrapped submanifold in $\mathbb{R}^{n+1,1}$. Then

$$
\left|\lambda_{1}\left(D_{\Sigma}\right)\right| \geq \frac{1}{2} \inf _{\Sigma}|\mathscr{H}|
$$


Moreover equality occurs if and only if $\Sigma$ is a totally umbilical round sphere in a spacelike hyperplane of $\mathbb{R}^{n+1,1}$.

Proof. It is enough to apply the previous theorem to an eigenspinor for $\not D$ associated with the eigenvalue $\lambda_{1}(\not D)$, and we directly have the result. From Theorem 14, $\Sigma$ lies in a totally geodesic spacelike hyperplane $P^{n+1}$ with constant positive mean curvature $H$. Then the Alexandrov theorem allows to conclude that $\Sigma$ is a totally umbilical sphere in $P^{n+1}$. The converse is clear by taking the restriction of a parallel spinor of the Minkowski space to $\Sigma$ via the totally geodesic immersion of $\mathbb{R}^{n+1}$ in $\mathbb{R}^{n+1,1}$.

\section{References}

[Almgren and Rivin 1998] F. J. Almgren, Jr. and I. Rivin, "The mean curvature integral is invariant under bending", pp. 1-21 in The Epstein birthday schrift, edited by I. Rivin et al., Geom. Topol. Monogr. 1, University of Warwick, Institute of Mathematics, Warwick, 1998. MR 2000a:53133 Zbl 0914.53007 arXiv math/9810183

[Andersson et al. 2011] L. Andersson, M. Eichmair, and J. Metzger, "Jang's equation and its applications to marginally trapped surfaces", pp. 13-45 in Complex analysis and dynamical systems IV, Part 2: General relativity, geometry, and PDE (Nahariya, 2009), edited by M. Agranovsky et al., Contemp. Math. 554, Amer. Math. Soc., Providence, RI, 2011. MR 2884392 Zbl 1235.53019

[Ballmann and Bär 2012] W. Ballmann and C. Bär, "Boundary value problems for elliptic differential operators of first order", pp. 1-78 in Algebra and geometry: in memory of C. C. Hsiung (Bethlehem, PA, 2010), edited by H.-D. Cao and S.-T. Yau, Surv. Differ. Geom. 17, International Press, Boston, 2012. MR 3076058

[Bär 1998] C. Bär, "Extrinsic bounds for eigenvalues of the Dirac operator", Ann. Global Anal. Geom. 16:6 (1998), 573-596. MR 99k:58183 Zbl 0921.58065

[Bartnik and Chruściel 2005] R. A. Bartnik and P. T. Chruściel, "Boundary value problems for Diractype equations", J. Reine Angew. Math. 579 (2005), 13-73. MR 2005k:58040 Zbl 1174.58305

[Bartnik and Simon 1982] R. A. Bartnik and L. Simon, "Spacelike hypersurfaces with prescribed boundary values and mean curvature", Comm. Math. Phys. 87:1 (1982), 131-152. MR 84j:58126 Zbl 0512.53055

[Baum et al. 1990] H. Baum, T. Friedrich, R. Grunewald, and I. Kath, Twistor and Killing spinors on Riemannian manifolds, Seminarberichte 108, Humboldt Universität, Sektion Mathematik, Berlin, 1990. MR 92h:53055 Zbl 0705.53004

[Benedetti and Silhol 1995] R. Benedetti and R. Silhol, "Spin and Pin" structures, immersed and embedded surfaces and a result of Segre on real cubic surfaces", Topology 34:3 (1995), 651-678. MR 96f:57033 Zbl 0996.57519

[Booß-Bavnbek and Wojciechowski 1993] B. Booß-Bavnbek and K. P. Wojciechowski, Elliptic boundary problems for Dirac operators, Birkhäuser, Boston, 1993. MR 94h:58168 Zbl 0797.58004

[Brown and York 1993] J. D. Brown and J. W. York, Jr., "Quasilocal energy and conserved charges derived from the gravitational action”, Phys. Rev. D (3) 47:4 (1993), 1407-1419. MR 94h:83046

[Bureš 1993] J. Bureš, "Dirac operators on hypersurfaces", Comment. Math. Univ. Carolin. 34:2 (1993), 313-322. MR 95g:58215 Zbl 0781.53031

[Eichmair et al. 2012] M. Eichmair, P. Miao, and X. Wang, "Extension of a theorem of Shi and Tam", Calc. Var. Partial Differential Equations 43:1-2 (2012), 45-56. MR 2012m:53075 Zbl 1238.53025 
[Hass and Hughes 1985] J. Hass and J. Hughes, "Immersions of surfaces in 3-manifolds", Topology 24:1 (1985), 97-112. MR 87a:57037 Zbl 0527.57020

[Herglotz 1943] G. Herglotz, "Über die Starrheit der Eiflächen”, Abh. Math. Sem. Hansischen Univ. 15 (1943), 127-129. MR 7,322a Zbl 0028.09401

[Hijazi and Montiel 2014] O. Hijazi and S. Montiel, "A holographic principle for the existence of parallel spinor fields and an inequality of Shi-Tam type", Asian J. Math 18:3 (2014), 489-506.

[Hijazi et al. 2001a] O. Hijazi, S. Montiel, and X. Zhang, "Dirac operator on embedded hypersurfaces”, Math. Res. Lett. 8:1-2 (2001), 195-208. MR 2002j:53053 Zbl 0988.53019

[Hijazi et al. 2001b] O. Hijazi, S. Montiel, and X. Zhang, "Eigenvalues of the Dirac operator on manifolds with boundary", Comm. Math. Phys. 221:2 (2001), 255-265. MR 2002g:58051 Zbl 0997.58015

[Hijazi et al. 2002] O. Hijazi, S. Montiel, and X. Zhang, "Conformal lower bounds for the Dirac operator of embedded hypersurfaces”, Asian J. Math. 6:1 (2002), 23-36. MR 2003i:58058 Zbl 1016.58013

[Jang 1978] P. S. Jang, "On the positivity of energy in general relativity”, J. Math. Phys. 19:5 (1978), 1152-1155. MR 80b:83012a

[Lam 2011] G. Lam, The graph cases of the Riemannian positive mass and Penrose inequalities in all dimensions, thesis, Duke University, Durham, NC, 2011, Available at http://www.math.duke.edu/ bray/PhD\%20Theses/Lam.pdf.

[Lawson and Michelsohn 1989] H. B. Lawson, Jr. and M.-L. Michelsohn, Spin geometry, Princeton Mathematical Series 38, Princeton University Press, 1989. MR 91g:53001 Zbl 0688.57001

[Liu and Yau 2003] C.-C. M. Liu and S.-T. Yau, "Positivity of quasilocal mass", Phys. Rev. Lett. 90:23 (2003), Article ID \#231102. MR 2004g:83031 Zbl 1267.83028

[Liu and Yau 2006] C.-C. M. Liu and S.-T. Yau, "Positivity of quasi-local mass, II", J. Amer. Math. Soc. 19:1 (2006), 181-204. MR 2007f:83032 Zbl 1081.83008

[Miao et al. 2010] P. Miao, Y. Shi, and L.-F. Tam, "On geometric problems related to Brown-York and Liu-Yau quasilocal mass", Comm. Math. Phys. 298:2 (2010), 437-459. MR 2011h:58022 Zbl 1200.58018

[Montiel and Ros 1997] S. Montiel and A. Ros, Curvas y superficies, Proyecto Sur, Armilla, 1997. Translated as Curves and surfaces, 2nd ed., Graduate Studies in Mathematics 69, Amer. Math. Soc., Providence, RI, 2009. MR 2010f:53007 Zbl 1075.53001

[Pinkall 1985] U. Pinkall, "Regular homotopy classes of immersed surfaces", Topology 24:4 (1985), 421-434. MR 87e:57028 Zbl 0583.57020

[Raulot 2013] S. Raulot, "The Dirac operator on untrapped surfaces", Comm. Math. Phys. 318:2 (2013), 411-427. MR 3020163 Zbl 06145993

[Rivin and Schlenker 1999] I. Rivin and J.-M. Schlenker, "The Schläfli formula in Einstein manifolds with boundary”, Electron. Res. Announc. Amer. Math. Soc. 5 (1999), 18-23. MR 2000a:53076 Zbl 0910.53036

[Schoen and Yau 1979] R. Schoen and S. T. Yau, "On the proof of the positive mass conjecture in general relativity”, Comm. Math. Phys. 65:1 (1979), 45-76. MR 80j:83024 Zbl 0405.53045

[Schoen and Yau 1981] R. Schoen and S. T. Yau, "Proof of the positive mass theorem, II", Comm. Math. Phys. 79:2 (1981), 231-260. MR 83i:83045 Zbl 0494.53028

[Shi and Tam 2002] Y. Shi and L.-F. Tam, "Positive mass theorem and the boundary behaviors of compact manifolds with nonnegative scalar curvature", J. Differential Geom. 62:1 (2002), 79-125. MR 2005b:53046 Zbl 1071.53018 
[Szabados 2004] L. B. Szabados, "Quasi-local energy-momentum and angular momentum in general relativity”, Living Rev. Relativity 7:4 (2004).

[Trautman 1995] A. Trautman, "The Dirac operator on hypersurfaces", Acta Phys. Polon. B 26:7 (1995), 1283-1310. MR 96m:58236 Zbl 0966.58504

[Wang and Yau 2007] M.-T. Wang and S.-T. Yau, "A generalization of Liu-Yau's quasi-local mass", Comm. Anal. Geom. 15:2 (2007), 249-282. MR 2008h:53046 Zbl 1171.53336

[Wang and Yau 2009] M.-T. Wang and S.-T. Yau, "Isometric embeddings into the Minkowski space and new quasi-local mass", Comm. Math. Phys. 288:3 (2009), 919-942. MR 2010d:53077 Zbl 1195.53039

[Weyl 1916] H. Weyl, "Über die Bestimmung einer geschlossenen konvexen Fläche durch ihr Linienelement”, Vierteljahrsschr. Naturf. Ges. (Zürich) 61 (1916), 40-72. JFM 46.1115.03

[Witten 1981] E. Witten, “A new proof of the positive energy theorem”, Comm. Math. Phys. 80:3 (1981), 381-402. MR 83e:83035 Zbl 1051.83532

[Yau 2001] S. T. Yau, "Geometry of three manifolds and existence of black hole due to boundary effect”, Adv. Theor. Math. Phys. 5:4 (2001), 755-767. MR 2003j:53052 Zbl 1019.53016

Received June 30, 2013. Revised September 17, 2013.

OUSSAMA HIJAZI

INSTITUT ÉLIE CARTAN DE LORRAINE

UNIVERSITÉ DE LORRAINE

NANCY I, Boîte Postale 239

54506 VAndoeuvre-LÈs-NANCY CEDEX

FRANCE

oussama.hijazi@univ-lorraine.fr

Sebastián Montiel

DEPARTAMENTO DE GEOMETRÍA Y TOPOLOGÍA

UNIVERSIDAD DE GRANADA

18071 GRANADA

SPAIN

smontiel@ugr.es

SiMON RAULOT

LABORATOIRE DE MATHÉMATIQUES RAPHAËL SALEM UMR 6085 CNRS

UNIVERSITÉ DE ROUEN AVENUE DE L'UNIVERSITÉ

BoÎte Postale 12 TECHNOPÔLE DU MADRILlet

76801 SAINT-ÉTIENNE-DU-ROUVRAY

FRANCE

simon.raulot@univ-rouen.fr 


\title{
PACIFIC JOURNAL OF MATHEMATICS
}

\author{
msp.org/pjm
}

Founded in 1951 by E. F. Beckenbach (1906-1982) and F. Wolf (1904-1989)

\section{EDITORS}

Don Blasius (Managing Editor)

Department of Mathematics

University of California

Los Angeles, CA 90095-1555

blasius@math.ucla.edu

\author{
Paul Balmer \\ Department of Mathematics \\ University of California \\ Los Angeles, CA 90095-1555 \\ balmer@math.ucla.edu \\ Robert Finn \\ Department of Mathematics \\ Stanford University \\ Stanford, CA 94305-2125 \\ finn@math.stanford.edu \\ Sorin Popa \\ Department of Mathematics \\ University of California \\ Los Angeles, CA 90095-1555 \\ popa@math.ucla.edu
}

\author{
Vyjayanthi Chari \\ Department of Mathematics \\ University of California \\ Riverside, CA 92521-0135 \\ chari@math.ucr.edu \\ Kefeng Liu \\ Department of Mathematics \\ University of California \\ Los Angeles, CA 90095-1555 \\ liu@math.ucla.edu \\ Jie Qing \\ Department of Mathematics \\ University of California \\ Santa Cruz, CA 95064 \\ qing@ cats.ucsc.edu
}

\section{PRODUCTION}

Silvio Levy, Scientific Editor, production@msp.org

\section{SUPPORTING INSTITUTIONS}

ACADEMIA SINICA, TAIPEI

CALIFORNIA INST. OF TECHNOLOGY

INST. DE MATEMÁTICA PURA E APLICADA

KEIO UNIVERSITY

MATH. SCIENCES RESEARCH INSTITUTE

NEW MEXICO STATE UNIV.

OREGON STATE UNIV.

\author{
STANFORD UNIVERSITY \\ UNIV. OF BRITISH COLUMBIA \\ UNIV. OF CALIFORNIA, BERKELEY \\ UNIV. OF CALIFORNIA, DAVIS \\ UNIV. OF CALIFORNIA, LOS ANGELES \\ UNIV. OF CALIFORNIA, RIVERSIDE \\ UNIV. OF CALIFORNIA, SAN DIEGO \\ UNIV. OF CALIF., SANTA BARBARA
}

\author{
Daryl Cooper \\ Department of Mathematics \\ University of California \\ Santa Barbara, CA 93106-3080 \\ cooper@math.ucsb.edu \\ Jiang-Hua Lu \\ Department of Mathematics \\ The University of Hong Kong \\ Pokfulam Rd., Hong Kong \\ jhlu@maths.hku.hk \\ Paul Yang \\ Department of Mathematics \\ Princeton University \\ Princeton NJ 08544-1000 \\ yang@math.princeton.edu
}

These supporting institutions contribute to the cost of publication of this Journal, but they are not owners or publishers and have no responsibility for its contents or policies.

See inside back cover or msp.org/pjm for submission instructions.

The subscription price for 2014 is US $\$ 410 /$ year for the electronic version, and \$535/year for print and electronic.

Subscriptions, requests for back issues and changes of subscribers address should be sent to Pacific Journal of Mathematics, P.O. Box 4163, Berkeley, CA 94704-0163, U.S.A. The Pacific Journal of Mathematics is indexed by Mathematical Reviews, Zentralblatt MATH, PASCAL CNRS Index, Referativnyi Zhurnal, Current Mathematical Publications and Web of Knowledge (Science Citation Index).

The Pacific Journal of Mathematics (ISSN 0030-8730) at the University of California, c/o Department of Mathematics, 798 Evans Hall \#3840, Berkeley, CA 94720-3840, is published twelve times a year. Periodical rate postage paid at Berkeley, CA 94704, and additional mailing offices. POSTMASTER: send address changes to Pacific Journal of Mathematics, P.O. Box 4163, Berkeley, CA 94704-0163.

PJM peer review and production are managed by EditFLOW ${ }^{\circledR}$ from Mathematical Sciences Publishers.

\section{PUBLISHED BY}

\section{mathematical sciences publishers \\ nonprofit scientific publishing}

http://msp.org/

(C) 2014 Mathematical Sciences Publishers 


\section{PACIFIC JOURNAL OF MATHEMATICS}

Volume $272 \quad$ No. $1 \quad$ November 2014

Nonconcordant links with homology cobordant zero-framed surgery $\quad 1$ manifolds

JAE CHOON CHA and MARK POWELL

Certain self-homotopy equivalences on wedge products of Moore spaces

Ho Won CHOI and KeE Young LeE

Modular transformations involving the Mordell integral in Ramanujan's lost notebook

Youn-SEO CHOI

The $D$-topology for diffeological spaces

J. DANiEl Christensen, Gordon SinNAMON and ENXIN Wu

On the Atkin polynomials

AHMAD EL-Guindy and Mourad E. H. ISMAIL

Evolving convex curves to constant-width ones by a perimeter-preserving flow

LAIYUAN GaO and Shengliang PAN

Hilbert series of certain jet schemes of determinantal varieties

SudhiR R. GHORPADE, Boyan Jonov and B. A. SeTHuraman

On a Liu-Yau type inequality for surfaces

Oussama Hijazi, Sebastián Montiel and Simon Raulot

Nonlinear Euler sums

ISTVÁN MEZŐ

Boundary limits for fractional Poisson $a$-extensions of $L^{p}$ boundary functions 227 in a cone

LEI QIAO and TAO ZHAO

Jacobi-Trudi determinants and characters of minimal affinizations

STEVEN V SAM

Normal families of holomorphic mappings into complex projective space concerning shared hyperplanes

LiU Yang, Caiyun Fang and Xuecheng Pang 